3. Wheeler, P.E. J. Hum. Evoln 13, 91 (1984)

4. Macfarlane, W.V. in Adaptations of Domestic Animal ed. Hafez, E.) 164-182 (Lea and Febifer, Philadelphia, 1968).

Montagna, W. in Biological Anthropology (ed. Katz, S.H.) 341-351 (Freeman, San Fransisco, 1975).

6. MacFarland, W.N., Pough, F.H., Cade, T.J. \& Heiser, J.B. Vertebrate Life, 674 (Collier Macmillan, London, 1979).

7. McFarland, D. Animal Behaviour, 267 (Pitman, London, 1985).

8. Schmidt-Nielsen, K. Desert Animals, 67 (Dover Publications, New York, 1979)

9. Hardy, A.C. New Scient. 7, 642 (1960)

10. Morgan, E. The Aquatic Ape (Souvenir, London, 1982).

11. Verhaegen, M.J.B. Med. Hypotheses 16, 17 (1985)

\section{Divergent genetic codes}

SIR-A recent News and Views article by Grivell', commenting on a paper by Hanyu et al. ${ }^{2}$, and an earlier commentary by $\mathrm{Fox}^{3}$ both addressed evidence for divergence from the universality of the genetic code in some ciliated protozoa. These organisms apparently use UAA (ochre) and UAG (amber), which are normally stop codons, to encode glutamine in polypeptides. Hanyu et al. provide evidence for the existence of two unusual glutamine tRNAs in Tetrahymena thermophila, one of which recognizes both UAA and UAG codons in messenger RNAs, whereas the other recognizes only UAG codons. In considering the evolution of tRNAs which can use UAA and UAG as sense codons in polypeptides, Hanyu et al. suggest "the stop codons UAA and UAG were rarely used during prociliate evolution and that weak suppressor tRNAs existed for these codons very early. Spontaneous point mutations creating UAA and UAG codons within protein genes would then not have been lethal for these prociliates . . . Once those early suppressor tRNAs acquired anticodons for perfect UAA and UAG recognition, respectively, the use of these codons as amino-acid codons became fixed". We would like to draw attention to supporting evidence in the form of weak amber and ochre suppressor tRNAs in yeast that are normally changed with glutamine.

We have shown that the yeast Saccharomyces cerevisiae contains two normal tRNA $^{\mathrm{Gln}}$ genes, one bearing the anticodon UUG and the other $\mathrm{CUG}^{4,5}$. When present on multicopy plasmids, the tRNA ${ }_{\text {GAA }}^{\text {Gln }}$ gene effects weak suppression of two different ochre mutations ${ }^{4}$, whereas the tRNA ${ }_{C A G}^{\mathrm{GIn}}$ gene suppresses a number of different amber mutations ${ }^{5}$. In both cases, codon-anticodon interactions between the nonsense codons and the tRNAs requires $U \cdot G$ mispairing at the first rather than the third codon position which may occur by weak first position wobble in organisms that use the conventional genetic code. More recently, by use of a strategy that involves deleting the single genomic copy of the essential yeast tRNA ${ }_{\text {CAC }}^{\mathrm{Gin}}$ gene and rescuing the potential lethality resulting from the deletion by transforming cells with a multicopy plasmid carrying the tRNA ${ }_{\text {CAA }}^{\mathrm{Gln}}$ gene, we have shown that suppression of UAG codons can even result from a single copy of a tRNA ${ }_{C A G}^{G l n}$ gene, thus strengthening the physiological relevance of this phenomenon as an evolutionary mechanism for divergence of the genetic code (W.A.W., I Edelman, M.R Culbertson and E.C.F., unpublished data).

Based on established rules for codonanticodon paring we hypothesize that the evolutionary progenitors of the ciliated protozoa under consideration might possess tRNAs with the conventional anticodons UUG and CUG, which can decode the universal glutamine codons CAA and CAG. Random mutations in regions of these tRNAs outside of the anticodons could be readily tolerated during evolution, as there is no compelling a priori reason for their counter-selection. Given the precedent for limited readthrough of UAA and UAG codons in yeast ${ }^{4,5}$, we argue that altered glutamine $\mathrm{tRNAs}$ with enhanced stability for first codon position wobble might in fact be strongly favoured in organisms in which the stop codons UAA and UAG had entered the coding region of essential genes by random genetic drift. Selection for these tRNAs could be facilitated by other mutational changes which further enhance the stability of first codon position wobble, for example alterations in ribosome binding and modification of bases in and/or outside the anticodon. Such evolutionary selection could eventually result in highly efficient suppression of these 'stop' codons, resulting in their retention in the coding region of genes of ciliated protozoa. This model is consistent with the fact that Tetrahymena thermophila contains a family of cognate tRNAs, some of which can decode CAA and/or UAA codons and others of which can decode CAG and/or UAG codons ${ }^{2}$.

Grivell also speculates on codon recognition by glutamine tRNA species in organisms which branched off the main line of eukaryotic evolution before or about the same time as the ciliates. He notes that Dictyostelium discoideum and Trypanosoma brucei (both of which branched off before the ciliates) show no deviation from the standard genetic code. According to some studies, the yeast $S$. cerevisiae may have branched just after the ciliates, with a much closer interval between the ciliates and yeast than that between the ciliates and $D$. discoideum or $T$. brucei ${ }^{6}$. Our observation ${ }^{1}$ that $S$. cerevisiae shows the vestiges of "an intrinsic property of a primitive or error-prone code" supports the hypothesis that misreading of nonsense codons by normal glutamine tRNAs (which has been preserved in yeast) may have preceded the use of UAA and UAG as sense codons for glutamine.

ERrol C. FriedBerg William A. Weiss

Department of Pathology,

Stanford University School of Medicine, Stanford, California, 94305, USA
Grivell, L.A. Nature, 324, 109-110(1986)

Hanyu, N. et al. EMBO J. 5, 1307-1311 (1986)

Fox, T.D. Nature 314, 132-133 (1985)

Pure, G.A. et al. J. molec. Biol, 183, 31-42 (1985)

Weiss, W.A. \& Friedberg, E.C. J. molec. Biol. (in the

M.L. et al. Proc. natn. Acad. Sci. U.S.A. 83, 1383 1387 (1986)

\section{Mystery object identified?}

SiR-Artefacts closely resembling the mystery object recently encountered by Wolstenholme et al. (Nature 323, 300; 1986) in a chromosome preparation are routinely fabricated in electronics laboratories by means of positive-resist electron-beam lithography. During the fabrication of certain semiconductor devices, a layer of poly[methyl methacrylate] (called PMMA) about 0.5 to $1 \mu \mathrm{m}$ thick is deposited on a silicon wafer. The PMMA layer is irradiated in a given pattern with an electron beam having a spot size as low as $0.01 \mu \mathrm{m}$ in diameter. The irradiated areas, being of reduced molecular weight, are then dissolved in a solvent that attacks the lower-molecular-weight areas. This leaves a pattern of holes in the PMMA layer; such patterns have resolutions of $0.1 \mu \mathrm{m}$ or better. Normally the remaining PMMA pattern is dissolved by a solvent during subsequent processing steps. If in the course of experimentation the wafer is discarded before the PMMA pattern is dissolved, it is possible for the pattern to peel off the wafer and enter the environment as a dust particle.

It does not strain credulity to consider the mystery object as a fragment of a PMMA pattern that has contaminated the chromosome preparation. A regular grid of squares of the size suggested by the photograph of Wolstenholme et al. is a useful test pattern for experiments in semiconductor device fabrication techniques; the 'black' squares in the photograph are probably air bubbles.

W.F. CLOCKSIN

Computer Laboratory,

University of Cambridge,

Corn Exchange Street,

Cambridge CB2 $3 Q G, U K$

WOLSTENHOLME REPLIES-In addition to the published 'explanations' of our 'mystery object', others have been sent directly to us. The majority of these suggest an extraterrestrial origin for the object but we prefer an explanation closer to home.

The various biological 'grids' that have been drawn to our attention - some of which are very beautiful - are never as regular as ours. We therefore prefer to believe that the object is man-made, although exactly what it is and how it arrived in our preparations we are still at a loss to understand. We agree that the black squares are probably air bubbles.

J. WOLSTENHOLME

Department of Medical Genetics,

Churchill Hospital,

Headington, Oxford $O X 37 L J, U K$ 